\title{
Portable and Non-invasive Blood Glucose Monitoring over a Prolonged Period using Whispering Gallery Modes at $2.4 \mathrm{GHz}$
}

Louis WY Liu, Khiem Nguyen Trong, Phuc Vo Hong, Phong Phan Thanh, Phuc Tran Hoc, Gioi Bui Manh, An Trieu Gia, Christian Richardson Escobia, Khoa Chu Dinh, Nguyen Hai Nam, Duong Minh Khoi, Dinh Quang Vinh, Nguyen Khanh Quynh, Do Nguyen Hoang, Nguyen Hong Lam Giang, Duong Thanh Minh, Nguyen Phan Bao Viet, Nguyen Tran Minh Luan, Nguyen Hoang Phuong, Le Thanh Tung Faculty of Engineering

Vietnamese German University

Thu Dau Mot City, Vietnam

liu.waiyip@vgu.edu.vn

\author{
Son Nguyen Thanh \\ Eastern International University \\ Eastern International University \\ Binh Duong New Township of Industry \\ Service and Residence \\ Vietnam \\ Liu Wai Shun \\ Maple Studio \\ Sheung Shui, NT., Hong Kong
}

\author{
Bal Virdee \\ Research Centre for Communications \\ Technology \\ School of Computing and Digital \\ London Metropolitan University \\ London, UK \\ Liu Yuen Lam \\ Maple Studio \\ Sheung Shui, NT., Hong Kong
}

\author{
Abhishek Kandwal \\ Chinese Academy of Sciences \\ China
}

Zoya Eremenko

O. Ya. Usykov Institute for

Radiophysics and Electronics

National Academy of Sciences of Ukraine

\begin{abstract}
Invasive measurement of blood glucose is not approprate for everyone, particularly the patients with leukemia. Here, we demonstrate how the blood glucose can be non-invasively monitored over a prolonged period in the absence of any expensive equipment. Method: A portable and non-invasive glucose sensor capable of monitoring blood glucose at real-time has been successfully constructed and tested in the absence of any vector network analyzer. Using vacuum suction, the sensor head of the proposed non-invasive glucose sensor forms a whispering gallery resonator out of a skin tissue on an arm during the measurement process. The architecture of the proposed glucose sensor is equipped with standard components, including a WIFI transmitter, an RSSI sensor and a microcontroller based computer display. Results: Using the proposed glucose sensor, a healthy volunteer has been his blood glucose levels monitored over 72 minutes after consuming a loaf of bread and a cup of cow milk. The measured blood glucose rose shortly after the meal until it peaked at 40 minutes and finally fell to the initial value at around 72 minutes. Conclusion: The overall results were in general consistent with the expected results. The proposed glucose sensor is expected to be instrumental for the individuals who dislike the traditional lancets.
\end{abstract}

Keywords-diabetics, blood glucose, Whispering Gallery modes, S-band, network analyzer, invasive blood glucose measurement, non-invasive measurement, Acuu-chek тм, RF/microwave, leaky waves

\section{INTRODUCTION}

Invasive measurement of blood glucose based on traditional lancets is not appropriate for everyone, particularly for the patients with leukemia. There have been incidents of Hepatitis $\mathrm{C}$ virus (HCV) infection due to the use of a capillary blood glucose meter (CBGM) lancet [6]. There is certainly a demand for non-invasive blood glucose sensor for home diagnosis of diabetics although non-invasive blood glucose sensor is hard to find not only in the market but also in published literature [3-5].

Non-invasive blood glucose monitoring at $\mathrm{RF} /$ microwave frequency is by far the most proven method of diagnosis [12]. Although the demand for non-invasive blood glucose sensor is high, design and construction of a reliable and accurate non-invasive blood glucose levels remain a challenge. The following reasons are:

1) At RF/microwave frequencies, the glucose sensor can act as a leaky wave antenna, which releases and absorbs leaky waves. The energy supposedly used for measurement at the receiving end of the sensor might have leaked as radiations to the surrounding, ending up with unstable and inaccurate blood glucose measurement.

2) The volume of blood to be measured is difficult to be ascertained because of the geometry and the non-invasive nature of a would-be non-invasive glucose sensor.

By simply suppressing leaky waves, we show that all these technical issues as mentioned above are the things of the past. Electromagnetic waves can be broadly divided into two kinds, surface waves and leaky waves. Leaky waves radiate to all directions, whilst surface waves are bound to an interface between two differing materials. Leaky waves are those radio waves used for radio communication, whilst surface waves spread over a surface. Leaky waves or radio waves tend to be unstable, whilst surface wave tend to be stable. The frequency at which a surface wave is transformed to a leaky wave is known as the cut-off frequency. At the frequencies below this cut-off frequency, the surface waves dominate the propagation modes, whilst the leaky waves are expected to be suppressed. By conducting the glucose measurement at frequencies below the cut-off frequency, we have successfully eliminated all the possibility of instability caused by leaky waves in the passage between the input and the output. The non-invasive glucose 
sensor to be proposed is based purely whispering gallery waves, which is a form of non-radiating surface waves.

The proposed portable glucose sensor is capable of noninvasively monitoring blood glucose of a period without any interruption. The total volume of blood to be monitored or measured is fixed through the formation of a whispering gallery mode resonator out of the human skin. The accuracy of the proposed sensor has been verified in several clinical trials held in Vietnamese-German University.

\section{METHOD OF SOLUTION}

The most important part of the proposed glucose sensor was the sensor head as shown in Fig. $1 \mathrm{~b}[1,2]$. The sensor head operates mainly on non-radiating surface waves. A source of surface waves is whispering gallery waves inside the tissue bump which was formed out of the skin tissue during the process of glucose measurement (See Fig. 1 b). Although formation of this tissue bump necessitate application of a suction pressure, this procedure is large non-invasive. In this work, this tissue bump was used as a whispering gallery resonator.

In theory, whispering gallery waves are formed as a result of successive internal reflections within a resonator. As illustrated in Fig. $1 \mathrm{~b}$, this so-called whispering gallery resonator has no sharp edge, thus favoring formation of whispering gallery waves. The vacuum suction has one tuning knot enabling the suction pressure or the thickness of this whispering gallery resonator to be manually controlled. This thickness is one of the parameter controlling the resonant whispering gallery resonant frequencies. Under a normal circumstance, the suction pressure does not need to be adjusted from experiment to experiment.

Another source of surface waves comes from the looped Goubau line winding the sensor head. The looped Goubau line is a transmission media supporting propagation of surface waves. This surface wave together with the whispering gallery waves within the tissue bump collectively resonates at certain frequencies known as whispering gallery resonant frequencies. The collective strength of these surface waves increase enormously during a whispering gallery resonance. When these surface waves become a dominant propagating mode, the leaky waves are largely suppressed, thus eliminating the likelihood of instably during the blood glucose measurement. In order to minimize the likelihood of instability due to the leaky waves, the blood glucose measurements were conducted at a frequency close to the fundamentally whispering gallery resonant frequency (i.e. $2.18 \mathrm{GHz})$.

When a blood glucose level changes, it induces a change in the permittivity of the blood. The imaginary part of the permittivity change will lead to a shift in the whispering gallery resonant frequency. This frequency shift will in turn be translated into a magnitude change in the transmission coefficient (i.e. S21). Fig. 1 a shows that there exists a highly linear correlation between the magnitude of the transmission coefficient and the fundamental whispering gallery resonant frequency.

Fig. 2 a shows the overall system architecture of the proposed portable glucose sensor. The sensor head is just one part of the overall system architecture. The sensor head has two ports, one of which is connected to the RF/microwave transmitter (see Fig.3) and the other connected to the radio signal strength indicator (i.e. RSSI). The RF/microwave transmitter is based on a WIFI generator of which the output is an RF signal at $2.4 \mathrm{GHz}$. Ahthough the sensor head resonates fundamentally at $2.18 \mathrm{GHz}$, the sensor head is expected to work equally well at $2.4 \mathrm{GHz}$ [1,2]; As Fig. 2 a illustrates, the $\mathrm{RF} /$ microwave transmitter was controlled through an Arduino board consisting of an Atmel 8-bit AVR microcontroller.

Connected at the receiving end is a radio signal strength indicator (RSSI). Instead of measuring the S21 parameter using a vector network analyzer, the S21 parameter of the looped Goubau line as a result of a blood glucose level was measured using the RSSI sensor. The strength of the signal received at the receiving end was converted into a $\mathrm{DC}$, which is then picked up by the acquisition circuit (Fig. 2 a).

The DC voltage from the RSSI device was very small, typically in the range of 100 microvolts. The acquisition circuit as shown in Fig. 2 a has a built-in amplifier amplifying the voltage to a readable value, say millivolt. The final voltage output from the acquisition circuit was continuously plotted on the computer screen using another microcontroller program written in $\mathrm{C \#}$. With the $\mathrm{C \#}$ program, the microcontroller continuously captures the blood glucose level in the form of a received DC voltage and displayed in the computer.

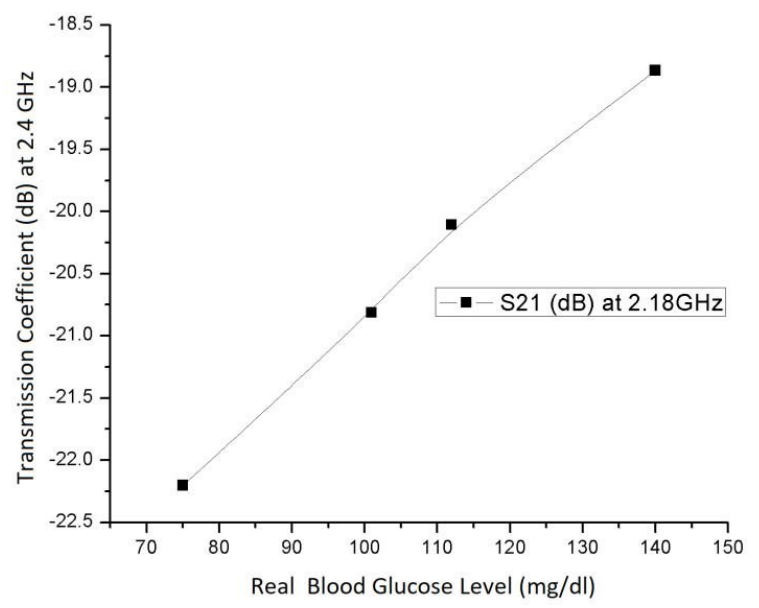

(a)

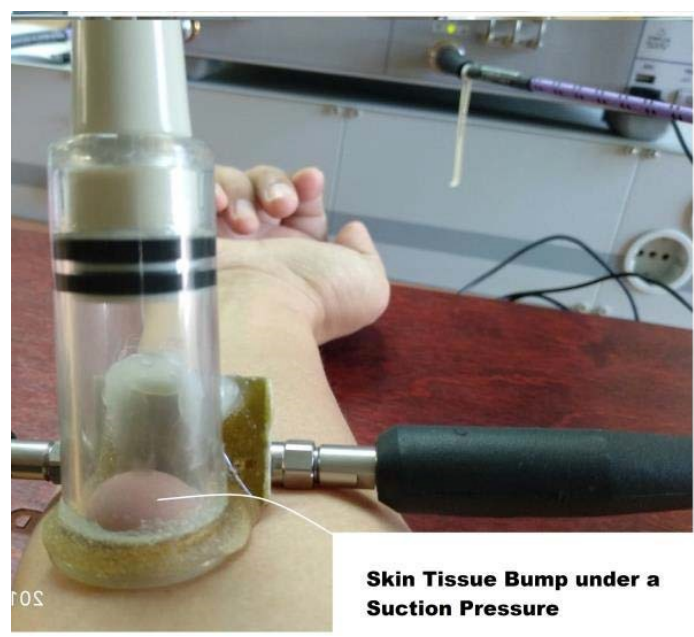

(b)

Fig. 1. Sensor head a) The measured characteristic of the sensor head at $2.18 \mathrm{GHz}$, which also works at $2.4 \mathrm{GHz}[1,2]$; b) The photograph of the sensor head. 


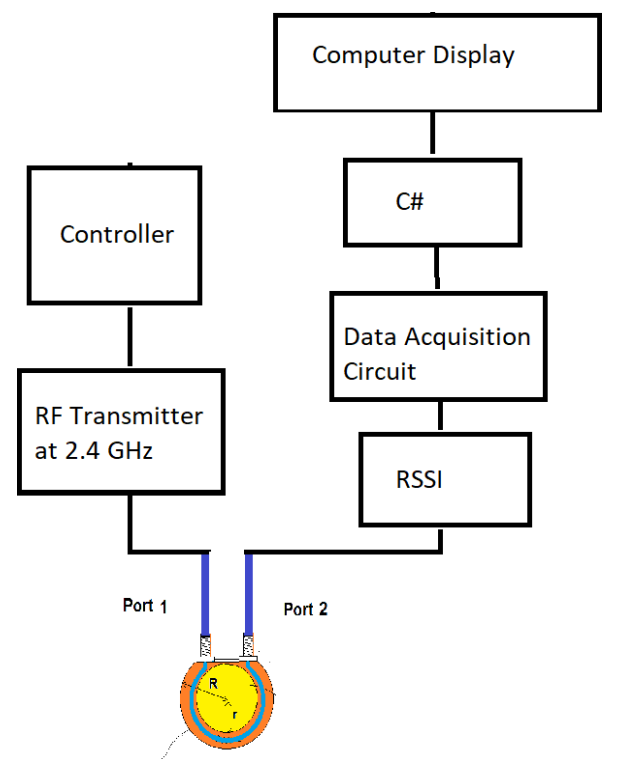

Sensor Head

(a)

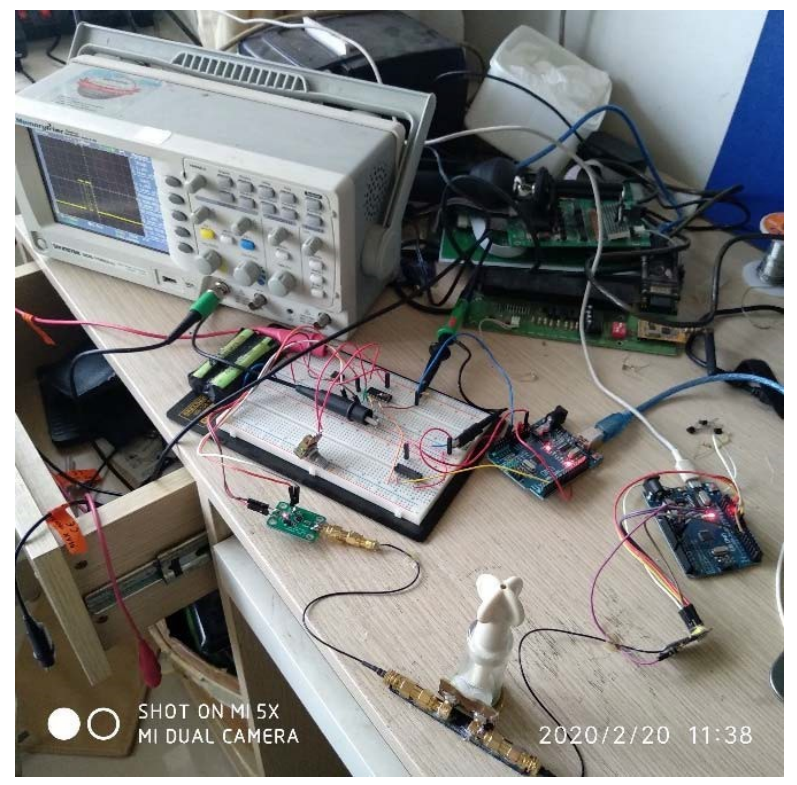

(b)

Fig. 2. The overall system architecture of the proposed portable glucose sensor. a) The schematic diagram; and b) The photograph illustrating the overall system.

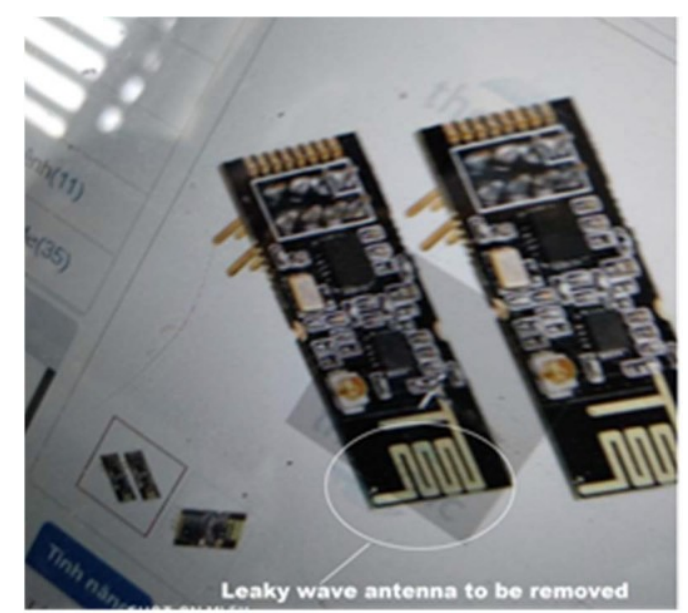

Fig. 3. RF/microwave Transmitter generating an RF at $2.4 \mathrm{GHz}$.

\section{EXPERIMENTAL RESULTS}

We have conducted a number of measurements focusing on non-fasting glucose changes. In one of the experiments, the volunteer was asked to use the proposed sensor to measure his blood glucose for one hour following consumption of a starchpacked small meal. The experimental process is shown in Fig 4. Being low in fiber, the meal contained one slide of white bread and a cup of cow milk. The blood glucose rose and fell in a noticeably manner as predicted by conventional approach. The initial blood glucose level of the volunteer was $70 \mathrm{mg} / \mathrm{dl}$ before meal. As shown in Fig. 5, the blood glucose rose steadily until it reached 40 minutes. After the initial 40 minute period, the blood glucose fell down until it reached the initial value at 72 minutes. This 72 minute period is believed to be the total time of chemical digestion in the body.

In the second meal, the volunteer ate some rice together with some protein based food, the peak time occurred at 55 minutes and the total time of chemical digestion was around 120 minutes.

According to our measurements, the volunteer usually felt hungry and cranky whenever his blood glucose level came crashing back down abruptly.

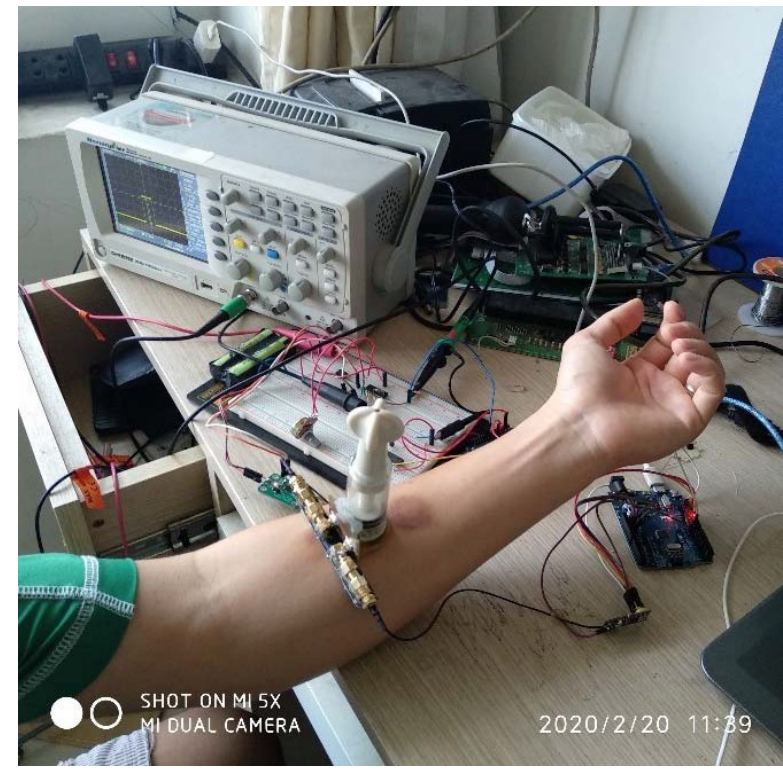

Fig. 4. Measurement setup using the proposed glucose sensor.

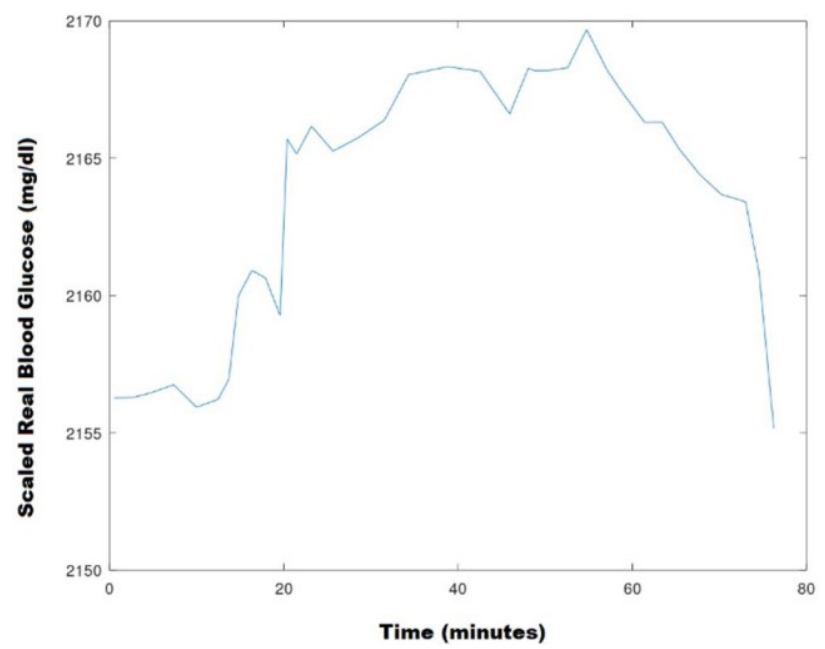

Fig. 5. Scaled blood glucose obtained by measured using the proposed sensor. 


\section{DISCUSSION}

The overall system has proven functional as expected. As predicted, the blood glucose as measured by the proposed portable glucose sensor rose initially until it peaked at around 40 minutes from the start. The blood glucose finally fell to the initial value at around 72 minutes from the start. This 72 minute period is the total time required for chemical digestion of the meal, which includes a slide of white bread and a cup of cow milk.

The total time required for chemical digestion depends on the type of food and the amount of the food. Protein based foods are known to be difficult to digest in the body. The total time taken to digest the protein was around 120 minutes, whilst the typical period is normally anywhere between 100 minutes and 120 minutes. The measured total time of digestion for the second meal was clearly consistent with the norm. The overall results were in general consistent with the results from some other research groups [6].

As Fig. 1a suggests, the transmission coefficient (i.e. S21) of the sensor head is almost proportional to the real blood glucose level. This characteristic was obtained from an in-vivo experiment when the operating frequency was around 2.18 $\mathrm{GHz}[2]$. The accuracy of the sensor head already been proven by several experiments using the vector network analyzer, which is an expensive piece of equipment $[1,2]$. At the time of this writing, the cost of an RS vector network analyzer is around US $\$ 70,000$. Instead of using a vector network analyzer for monitoring blood glucose, as was done in other methods [7], we have used a Radio Signal Strength Indicator (RSSI) to measure the transmission efficiency. This transmission efficiency should be very close to S21 if and only if the antenna effects or the leaky wave radiation losses were minimal.

The glucose over time plot as shown in Fig. 5 was not very smooth, in part because of the resolution problem of the RSSI sensor. The glucose over time can be further curve-fitted, depending on the applications.

The glucose concentration change in the blood can yield both a magnitude change in S21 and resonant frequency shift in the S21 graph. The above methodology has used the magnitude change of the measured S21 parameters to determine the blood glucose levels. In another experiment conducted using the same sensor, we have monitored the resonant frequency shift to determine the blood glucose levels. The frequency shift approach was found to be superior in terms of specificity in the sense that the measured results were almost independent of the effects of the contaminants on the skin tissue and the electromagnetic disturbance from the surrounding environment. If this is the case,

\section{CONCLUSION}

We have constructed a non-invasive glucose sensor capable of monitoring blood glucose at real-time in the absence of any vector network analyzer, which is an expensive equipment. The realized non-invasive glucose sensor is perfectly portable. The proposed sensor was based on a sensor head capable of forming a whispering gallery resonator out of a skin tissue on an arm using a vacuum suction pressure. Instead of relying on a vector network analyzer, or any other expensive microwave equipment, the architecture of the proposed glucose sensor consists of a WIFI transmitter (a signal source generating an RF at $2.4 \mathrm{GHz}$ ), an RSSI sensor and a microcontroller based computer display. The measured change in the glucose level in the blood was expected to cause a change in both S21 magnitude and the resonant frequency. In this work, the S21 magnitude change was used to measure the change in the blood glucose level. Using the proposed glucose sensor, the blood glucose of a healthy volunteer has been monitored over 72 minutes after the volunteer has eaten a meal containing a loaf of bread and a cup of cow milk. The measured blood glucose rose shortly after the meal until it peaked at 40 minutes. The blood glucose finally fell to the initial value at 72 minutes. The overall results were in general consistent with the results from some other research groups. The proposed glucose sensor is expected to be instrumental for the individuals who cannot tolerate the pain following the use of traditional lancets.

\section{ACKNOWLEDGMENT}

The clinical trials of this work were conducted by the volunteers who have agreed to do the experiments in writing. Son and Liu share equal contribution to this work.

\section{REFERENCES}

[1] Louis WY Liu, Abhishek Kandwal, Qingsha Cheng, Hongjian Shi, Igbe Tobore and Zedong Nie, "Non-Invasive Blood Glucose Monitoring Using a Curved Goubau Line", Electronics 2019, 8(6), 662; https://doi.org/10.3390/electronics8060662

[2] Louis W. Y. Liu, Abhishek Kandwal, A.E. Kogut, Zoya Eremenko, E. Kogut, Mohammad Tariqul Islam, R. S. Dolia, O.Ya.Usikov, S. Nosatiuk, Son T. Nguyen, "In-Vivo and Ex-Vivo Measurements of Blood Glucose Using Whispering Gallery Modes", Sensors 20(3):830, February 2020, DOI: https://doi.org/10.3390/s20030830

[3] K. Tachibana, K. Okada, R. Kobayashi and Y. Ishihara, "Development of a high-sensitivity and portable cell using Helmholtz resonance for noninvasive blood glucose-level measurement based on photoacoustic spectroscopy," 2016 38th Annual International Conference of the IEEE Engineering in Medicine and Biology Society (EMBC), Orlando, FL, 2016, pp. 6477-6480.

[4] E. Nakamachi, "Development of automatic operated blood sampling system for portable type Self-Monitoring Blood Glucose device," 2010 Annual International Conference of the IEEE Engineering in Medicine and Biology, Buenos Aires, 2010, pp. 335-338.

[5] M. S. Asekar, "Development of Portable Non-Invasive Blood Glucose Measuring Device Using NIR Spectroscopy," 2018 Second International Conference on Intelligent Computing and Control Systems (ICICCS), Madurai, India, 2018, pp. 572-575

[6] Faisal Inayat, MBBS and Aitzaz BinSultan Rai, MD, MSc (Oxon), "Acute hepatitis $\mathrm{C}$ virus infection related to capillary blood glucose meter", Saudi Med J. 2016 Jan; 37(1): 93-95. doi: $10.15537 / \mathrm{smj} .2016 .1 .13143$

[7] Kandwal, A., Igbe, T., Li, J. et al. Highly Sensitive Closed Loop Enclosed Split Ring Biosensor With High Field Confinement for Aqueous and Blood-Glucose Measurements. Sci Rep 10, 4081 (2020). https://doi.org/10.1038/s41598-020-60806-9. 